\title{
Coded wavefront sensor and non-iterative learning-based reconstruction
}

\author{
Miao Qi, Congli Wang, Wolfgang Heidrich \\ Visual Computing Center, King Abdullah University of Science and Technology, Thuwal 23955, Saudi Arabia \\ miao.qi@kaust.edu.sa
}

\begin{abstract}
Here we put forward a coded wavefront sensor and neural network framework to achieve wavefront reconstruction. The new framework outperform the preview method not only in the speed but also in the accuracy. (C) 2021 The Author(s)
\end{abstract}

\section{Introduction}

Wavefront sensor play an very important role in many research field. In biomedical imaging [1], optical aberration will happen when the light goes through the intracellular fluid material and some transparent tissue. The wavefront sensor plays an essential role in optical aberration correction and compensation. In the astronomical imaging [2], the atmosphere turbulence will cause some phase distortion, which will degrade the final image quality. We would introduce the adaptive optics to correct this distortion. One of the most important part of the adaptive optics is the wavefront sensor. It can detect the turbulence distortion and make the corresponding compensation . In the autonomous vehicle, it can help to correct some The wavefront sensor we used in this paper is the coded wavefront sensor [3]. We placed a binary code pattern in front of the camera. We first captured the image without any distortion as a reference. Then when the wavefront change, the distortion also changes. Then we can infer the wavefront from the distortion of the captured image and reference image.

Most of the preview wavefront sensor reconstruction methods are optimization-based iterative method [3], which need a very long time to handle the large image.

Because this inverse problem is under-determined, people add some hand-craft prior to solve it. However, firstly, design the hand-craft prior is always very time-consuming. Secondly, it is not easy to find an excellent hand-craft prior to fit all scenes and have a good reconstruction result. Recently, there is some advance in using deep learning in the phase retrieval problem. However, most of them are the iterative method. [4]

However, in this project, we put forward a deep learning non-iterative method. This can have better performance than the preview method not only in the speed but also in the accuracy.

\section{Principle}

\subsection{Coded Wavefront Sensor}

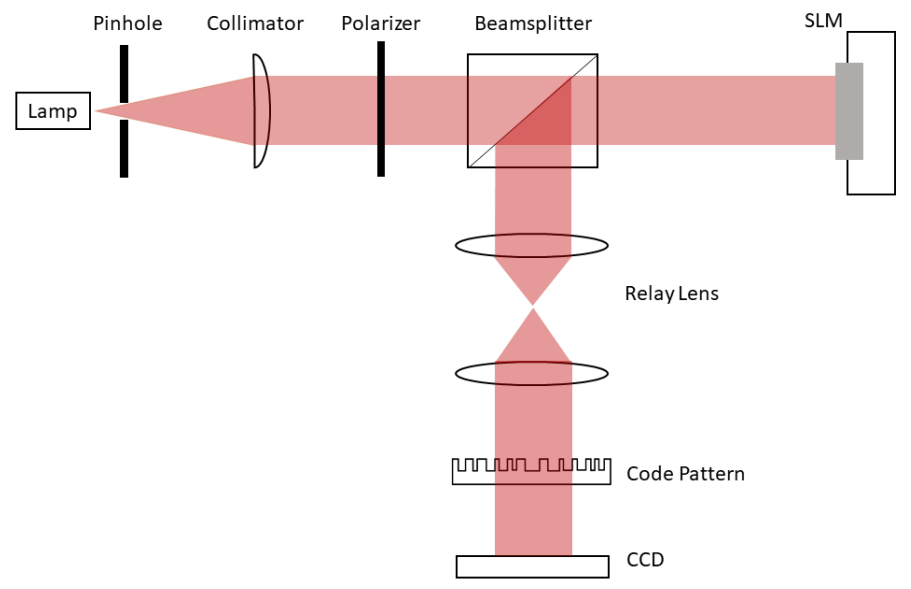

Fig. 1. Coded Wavefront Sensor 
The coded wavefront sensor is showing in Figure 1. We place a binary code pattern in front of the camera. The reference image $I_{0}$ is captured in a planar wavefront. When the object wavefront change, there is distortion. We capture another image $I$. We can infer the wavefront from the distortion. The whole process can be written as the following equation:

$$
\frac{z}{k} \nabla \phi(\mathbf{r}) \cdot \nabla \mathbf{I}_{\mathbf{0}}(\mathbf{r})+\mathbf{I}(\mathbf{r})-\mathbf{I}_{\mathbf{0}}(\mathbf{r})=\mathbf{0}
$$

$z$ is the distance between the mask camera. $k=2 \pi / \lambda$ is the wave number. The setup is shown in Figure 1 . The wavefront is displayed by the SLM.

\section{2. related work}

The Metzler proposed the prDeep unrolled neural network [4]. The author use the idea of the RED [5] and plugand-play prior [6]. In the plug-and-play prior paper, the author use the denoiser(like BM3D) as the projection operator for the prior sub-problem.

\subsection{Simulation and Reconstruction}

Because all the physical process is well-understood [7], we built a simulation code base on the Fast Fourier Transform for the wave propagate in the free space [8]. The input of simulation data is human bone captured by ptychography from [9]. The resolution is $256 \times 256$ grayscale image. The sensor pixel size is $6.45 \times 10^{-} 6$. The binary mask pattern is placed $1.5 \mathrm{~mm}$ before the CCD. The wave length is $550 \mathrm{~nm}$.

We built an attention UNet [10] for reconstruction. The attention UNet is made of encoder and decoder. It has good performance in this kind of image reconstruction task.

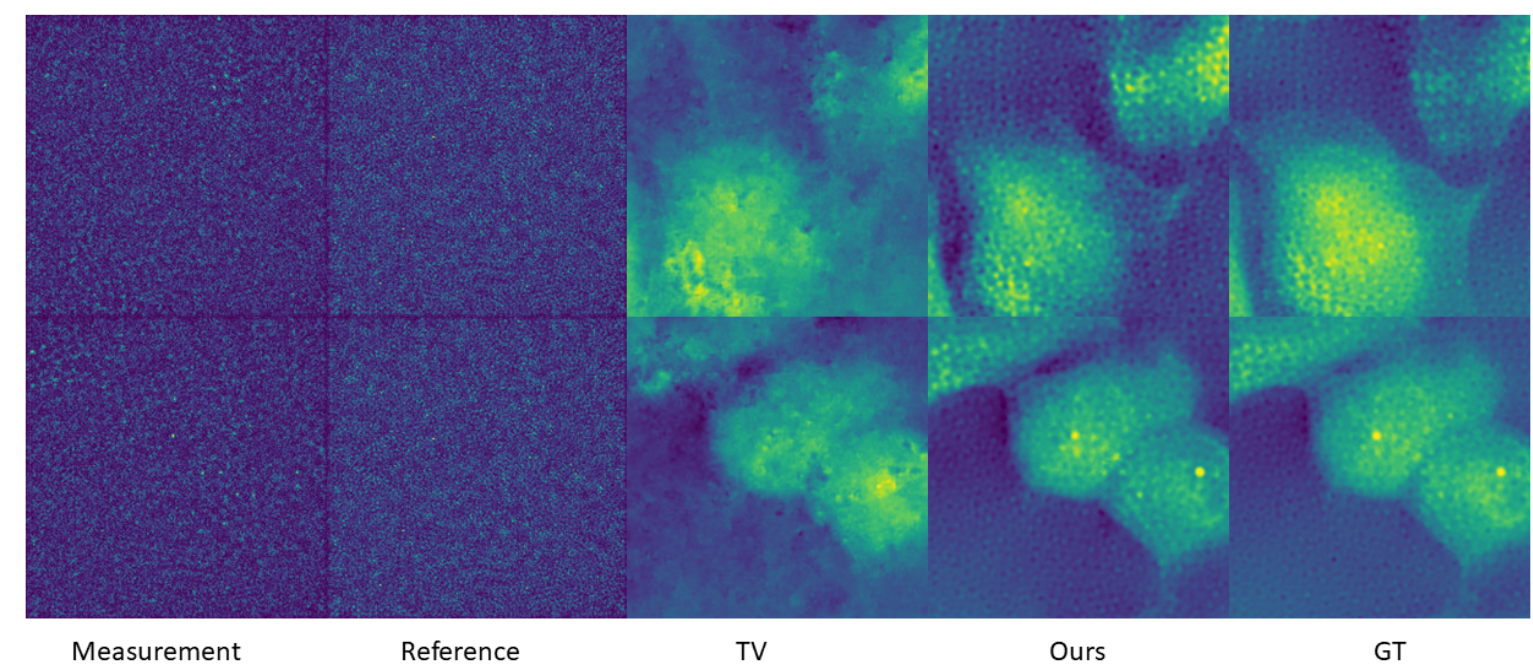

Fig. 2. Reconstruction Result Comparison

There is a lot of small structure in the biological imaging, which will be a challenging problem for the priorbased method. We can see from the Figure 2. The TV prior method result is very smooth. All the detailed structure is lost. Meanwhile, our deep learning method result can reconstruct even the small bubble in the image very well. The metrics result in our method outperform the TV-prior method by 9dB in PSNR and 0.4 in SSIM.

\section{Conclusion}

In this paper, we put forward a very simple wavefront measurement method based on the image's distortion. We tried a deep learning method for the reconstruction algorithm. The result outperforms the preview priorbased method. This enables such wavefront sensor to become practical in the biological image and nanostructure reconstruction task.

\section{References}

1. N. Ji, “Adaptive optical fluorescence microscopy," Nat. methods 14, 374-380 (2017).

2. R. K. Tyson, Principles of adaptive optics (CRC press, 2015). 
3. C. Wang, X. Dun, Q. Fu, and W. Heidrich, "Ultra-high resolution coded wavefront sensor," Opt. express 25, 1373613746 (2017).

4. C. Metzler, P. Schniter, A. Veeraraghavan et al., "prdeep: robust phase retrieval with a flexible deep network," in International Conference on Machine Learning, (PMLR, 2018), pp. 3501-3510.

5. Y. Romano, M. Elad, and P. Milanfar, "The little engine that could: Regularization by denoising (red)," SIAM J. on Imaging Sci. 10, 1804-1844 (2017).

6. S. V. Venkatakrishnan, C. A. Bouman, and B. Wohlberg, "Plug-and-play priors for model based reconstruction," in 2013 IEEE Global Conference on Signal and Information Processing, (IEEE, 2013), pp. 945-948.

7. J. W. Goodman, Introduction to Fourier optics (Roberts and Company Publishers, 2005).

8. K. Matsushima and T. Shimobaba, "Band-limited angular spectrum method for numerical simulation of free-space propagation in far and near fields," Opt. express 17, 19662-19673 (2009).

9. L. Tian, Z. Liu, L.-H. Yeh, M. Chen, J. Zhong, and L. Waller, "Computational illumination for high-speed in vitro fourier ptychographic microscopy,” Optica 2, 904-911 (2015).

10. O. Oktay, J. Schlemper, L. L. Folgoc, M. Lee, M. Heinrich, K. Misawa, K. Mori, S. McDonagh, N. Y. Hammerla, B. Kainz et al., "Attention u-net: Learning where to look for the pancreas," arXiv preprint arXiv:1804.03999 (2018). 